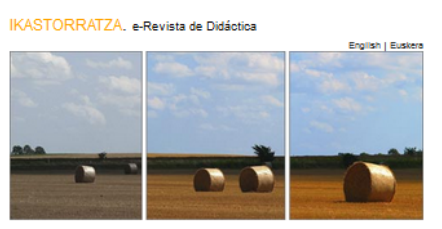

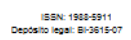

Inicio, Sobre nosotros, Publicacionesı, Participa

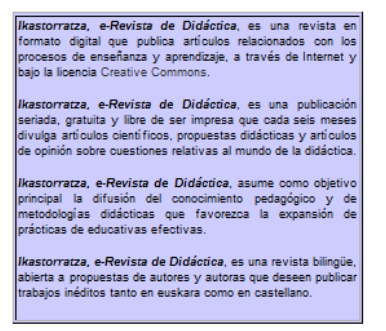

\section{IKASTORRATZA. Didaktikarako e-aldizkaria}

\author{
IKASTORRATZA. e-journal on Didactics
}

ISSN: 1988-5911 (Online) Journal homepage: http://www.ehu.eus/ikastorratza/

\title{
Apropiación de la escritura de los niños ikoots
}

Gervasio Montero Gutenberg monteroikoots@gmail.com

To cite this article:

Montero Gutenberg, G. (2020). Apropiación de la escritura de los niños ikoots. IKASTORRATZA. e-Revista de Didáctica, 25, 23-49. DOI: 10.37261/25_alea/2

To link to this article:

https://doi.org/10.37261/25_alea/2

Published online: 31 october 2020 


\title{
Apropiación de la escritura de los niños ikoots
}

\author{
Gervasio Montero Gutenberg \\ Escuela Normal Bilingüe e Intercultural de Oaxaca \\ monteroikoots@gmail.com
}

\section{Resumen}

Este artículo de investigación se centra en el análisis de la escritura de los niños ikoots 'huaves' de segundo grado de educación primaria bilingüe (7-8 años), hablantes de la lengua ombeayiüts como primera lengua y el español como segunda, pero alfabetizados en ésta última. La perspectiva que guía la investigación es de índole cualitativa. Los resultados muestran que el alumnado, en español, se encuentran en el nivel alfabético medio, que ya han superado la hipótesis de composición silábica consonante-vocal. Mientras que en ombeayiüts, se basan en transferencias lingüísticas para realizar la representación de los fonemas compartidos con el español. No muestran grandes dificultades con los fonemas de escritura estable, mientras que el conflicto aparece con los de escritura inestable y con los exclusivos del ombeayiüts.

Palabras clave: Escritura; Transferencia lingüística; Lengua ombeayiüts; Educación bilingüe.

\begin{abstract}
This investigation paper analyses the writing production of the ikoots children 'huaves' who are in the second grade of a bilingual elementary school. They are between seven and eight years old and speak ombeayiüts as their fist language and Spanish as their second language. However they are being literate in the second language. The work is from a quatitative perspective. The results show that the students are in an intermediate level in Spanish literacy and that they have passed the hypothesis of consonant-vowel syllabic composition. Meanwhile in ombeayiüts, they rely on linguistic transfer to represent the phonemes shared with Spanish. They don't show great difficulties with the phonemes of stable writing but the conflict appears with the phonemes of unstable writing and with the phonemes in ombeayiüts.
\end{abstract}

Key words: Writing; Linguistic transfer; Ombeayiüts language; Bilingual education. 


\section{Introducción}

En México la educación en contextos de los grupos indígenas ha estado ubicada históricamente entre dos polos en relación a los propósitos y métodos seguidos para la implementación de la educación bilingüe, Por ejemplo, en el caso de la enseñanza del español: la castellanización directa (programa de submersión) y la asimilación indirecta a través de diversos métodos bilingües de tipo transicional (Francis y Hamel, 1992).

Con la creación de la Dirección General de Educación Indígena (DGEI) en 1978 y posteriormente, en 2001 la Coordinación General de Educación Intercultural Bilingüe (CGEIB), se dieron pasos y pautas hacia la implementación de una educación bilingüe, primero bicultural posteriormente intercultural. En este caso el énfasis estaba en colocar en el centro de la enseñanza la primera lengua o lengua materna (L1) de los educandos con tendencia a un bilingüismo aditivo. Sin embargo, esto ha estado alejado de la realidad de muchas escuelas en escenarios indígenas. Por ejemplo, en 2016-2017 la producción de libros en lengua indígenas solo abarcó 38 de las 68 lenguas indígenas existentes y la enseñanza de la lengua indígena solamente se desarrolla como asignatura (INEE, 2017).

Lo anterior refleja la situación de implementación de una educación bilingüe en el territorio mexicano. Sin embargo, experiencias con relación a la enseñanza bilingüe coinciden en que no existe ninguna justificación académica o lingüística para la exclusión ni de la lengua materna ni del español en el aula de clases en ningún nivel (Cummins, 1983; Cummins, 2005; Krashen, 1999; Marsh, Pérez y Ráez, 2015; Pérez, 2012). En las regiones indígenas, sin embargo, continúan las prácticas de castellanización basadas, en la gran mayoría de los casos, en los libros de texto oficiales para niños hispanohablantes que apenas son aptos para una enseñanza del español como segunda lengua (L2).

Por otro lado, no todos los niños y niñas indígenas tienen la oportunidad de asistir a escuelas públicas en donde su lengua y su cultura sean los centros de la enseñanza, sean éstos del sistema general o del subsistema de educación indígena. Por tal razón, muchos en los primeros grados se alfabetizan exclusivamente en español, segunda lengua de los niños y de la cual sus conocimientos orales son casi nulos.

En algunos escenarios bilingües, la escuela toma disposiciones programáticas en cuanto al uso de las lenguas en la enseñanza de la lectura y escritura. Estas pueden ser desde la exclusión completa de la L1 del alumnado, hasta el uso tanto de la L1 como de la L2 de forma 
progresiva. No es el propósito del presente texto referenciar esta temática, sino enfocar sólo un aspecto de la cuestión: la apropiación de la escritura de los niños ikoots ${ }^{1}$ de segundo grado, hablantes de la lengua ombeayiüts ${ }^{2}$ como L1 y el español como L2.

El artículo aborda como objetivo general analizar la producción escrita de los niños ikoots de segundo grado en dos lenguas: español como L2, pero retomada como lengua de instrucción y enseñanza en el aula y el ombeayiüts como L1, relegado a lengua de aparición en ciertos momentos aislados en la enseñanza. Se parte de la premisa inicial que los educandos a pesar de que no son alfabetizados en su L1, realizan transferencias lingüísticas para la producción escrita en ombeayiüts.

De este modo, el texto aborda en primer lugar la escritura y la educación bilingüe. Seguidamente se presenta el contexto de la cultura ikoots donde se encuentra localizada la Escuela Primaria Bilingüe “Moisés Sáenz”, lugar en el que se realizó la investigación con niños de segundo grado. A continuación se muestra la perspectiva metodológica que guía el trabajo y el proceso de análisis de la producción escrita de los educandos. Posteriormente, se anuncia los resultados, donde se describe y analiza la escritura de los alumnos en las dos lenguas: español como su L2 y el ombeayiüts como L1. Y para terminar, se aborda las principales conclusiones del estudio.

\section{Escritura y educación bilingüe}

Como se sabe, el lenguaje escrito no es una traducción literal del lenguaje oral, no es una transcripción del habla (Halliday, 1989), ya que el primero es una función especial del lenguaje, mientras que la oralidad es algo natural, innata al ser humano (Vigil, 2004). Por eso se establece que la escritura, a diferencia del lenguaje oral, es producto consciente y creativo de los seres humanos, posee un carácter más abstracto que el lenguaje oral. O como dice Prado (2004), es un código escrito inventado por el ser humano para poder retener el acto comunicativo y, por consiguiente, fijar una lengua por medio de la escritura permite la ampliación de los parámetros lingüísticos a un ámbito más extenso del oral (Ong, 2016).

\footnotetext{
1 Término que significa 'nosotros', utilizado por los habitants de San Mateo del Mar para referirse a su cultura de pertenencia, que comúnmente se conoce como 'huave'.

2 Palabra cuyo significado hace alusión a ‘nuestra boca, nuestra voz’ que la comunidad de San Mateo del Mar usa para nombrar a la lengua que se habla en su territorio.
} 
El desarrollo escolar de la lengua indígena, máxime si hablamos del proceso de escritura, dependen del cruce de muchos factores como: del hábito, la necesidad, el prestigio y, sobre todo, del alfabeto y la normalización. El bilingüismo con la disminución de monolingües y niños hablantes, plantea un reto a la escuela indígena y a la escuela en general para este siglo. Porque en contextos indígenas la lengua materna no es utilizada para la comunicación escrita, por lo que parece que la enseñanza directa del español es la única opción práctica para muchos docentes. Mientras, el uso de la escritura en L1 se mantiene limitado por la disputa y desacuerdos en la normalización y estandarización del alfabeto.

Por ello, en el sistema educativo indígena mexicano el aprendizaje de la escritura en una lengua que no es la L1 del educando, por ejemplo aprender a escribir en español teniéndolo como L2, es una situación común. Sin embargo, investigaciones han remarcado el papel fundamental que posee el hecho de que en los sistemas de educación bilingües se aprenda a escribir primero a partir de la lengua materna, para ser la base y referencia para el aprendizaje de la L2, ya que, los niños bilingües aprenden mejor cuando han desarrollado por lo menos una lengua. No obstante, ambas lenguas se utilizan en el currículum y la eliminación de la lengua materna de los alumnos no es considerada como una condición para aprender otra (Cummins, 2001). Como señala Schmelkes (2002):

Sabemos que es conveniente enseñar a leer y a escribir en la lengua materna. Por evidente que esto resulta -no es posible aprender a leer y a escribir en una lengua que uno no entiende- los maestros en sus prácticas no siempre muestran compartir este principio. La segunda lengua puede comenzarse a enseñar oralmente, y una vez que se muestran avances en los aspectos receptivos y creativos de la lengua oral (comprensión y expresión) puede enseñarse en forma escrita (p.15).

Sin considerar que las lenguas indígenas, como señala Vigil (2004) y Schmelkes (2002), son netamente orales y ágrafas, es decir, originalmente no tienen escritura. Esta es, no tienen grafías especiales que se hayan diseñado por el mismo grupo indígena para realizar su impresión y representación. Aunque en años recientes, el Instituto Nacional de Lenguas Indígenas (INALI) ha llevado a cabo la producción de normas de escritura en algunas lenguas, el desarrollo o la enseñanza de la escritura en las escuelas ha sido limitada. Además, en muchos escenarios el proceso de apropiación de la escritura se da con base en el copiado 
o la transcripción de unidades gráficas, dejando de lado el proceso de construcción a partir del proceso psicogenético (Ferreiro y Teberovsky, 2007, Gómez, 2000, Nemirovsky, 1999, Ferreiro y Gómez, 1991).

En este sentido, dentro del proceso que existe entre leer y escribir y no poder hacerlo, existe un largo camino en el cual los niños poco a poco van conociendo y empleando las características del sistema de escritura. En una primera instancia, pueden ignorar que existe la relación entre la escritura y la oralidad, sin embargo, perciben que requieren de una cantidad mínima de grafías para que algo pueda ser legible (Ferreiro, 2004). Sin embargo, en el espacio escolar, este tipo de escritura en ocasiones pasa inadvertido por parte de los docentes, porque no se ha podido interpretar o analizar, a veces sólo se observan escrituras caóticas a primera vista, se les ha estigmatizado como carentes de significado, y que, para el profesorado, no cumple con las características de una escritura ya convencionalizada (Lerner, 2003).

Por lo tanto, la escritura al igual que la oralidad, comunica ideas y sentimientos, solo que la escritura el niño la considera compleja , ya que tiene que hacer una representación de fonemas y grafemas con los que no ha tenido relación, y si la ha tenido, aún no sabe qué representa cada carácter. De ello deriva que en este trabajo se aborde la apropiación de la escritura por parte de los niños y niñas ikoots. Se analiza cómo han avanzado en la construcción grafémica del español siendo su L2. a la vez, con base en transferencias lingüísticas, también muestran importantes logros en la graficación del ombeayiüts. La transferencia lingüística (TL) se entiende como la influencia resultante de las similitudes y diferencias entre la L1 y la lengua que se aprende (L2) (Odlin, 1989), pero para el caso que planteamos esto sucede al revés. Algunos estudios tratan la TL en diferentes niveles, desde el léxico y el morfosintáctico hasta el fonológico, considerando, generalmente, lo que hace el estudiante como resultado de la TL por instrucción del docente (Agustín, 2010; Odlin, 1989).

Para los propósitos de este estudio, la transferencia lingüística se entiende como la influencia que se detecta de la escritura de la L2 (lengua de instrucción y de enseñanza) en el uso y representación de algunos fenómenos de escritura en la L1. Se identifican y se analizan aspectos a partir de los fonemas que registran los educandos. 


\section{3. Área y contexto de estudio}

La investigación se centra en el municipio de San Mateo del Mar, población localizada en el sureste del estado mexicano de Oaxaca sobre el istmo de Tehuantepec a orillas del oceáno Pacífico (Figura 1), específicamente en Barrio Nuevo, con niños de segundo grado, grupo “A” de la Escuela Primaria Bilingüe "Moisés Sáenz”3. Los 24 niños son hablantes del ombeayiüts como L1, manteniendo el español como la L2. A pesar de ello, en el aula la lengua predominante es la L2, de modo que únicamente en momentos y situaciones aisladas se retoma la L1. Sin emabrgo, fuera del espacio áulico, los niños se comunican en su L1. El contexto forma parte de la cultura ikoots o más comúnmente conocida como 'huave', una de las 16 culturas que coexisten en el estado de Oaxaca. Sobre el origen del vocablo huave hay divergencias. Algunos autores y los habitantes mismos, consideran que es una denominación zapoteca de forma peyorativa y cuya referencia al castellano podría significar “podrido por la humedad, lodo podrido, o pies podridos”, dada la actividad que desempeñan (León, 1903, Signorini, 1991, Gómez, 2005). Por tal motivo, los miembros de la cultura principalmente los de San Mateo del Mar- han optado por autodefinirse como ikoots ‘nosotros’ y a la lengua que hablan ombeayiüts ‘nuestra boca, nuestra voz’.

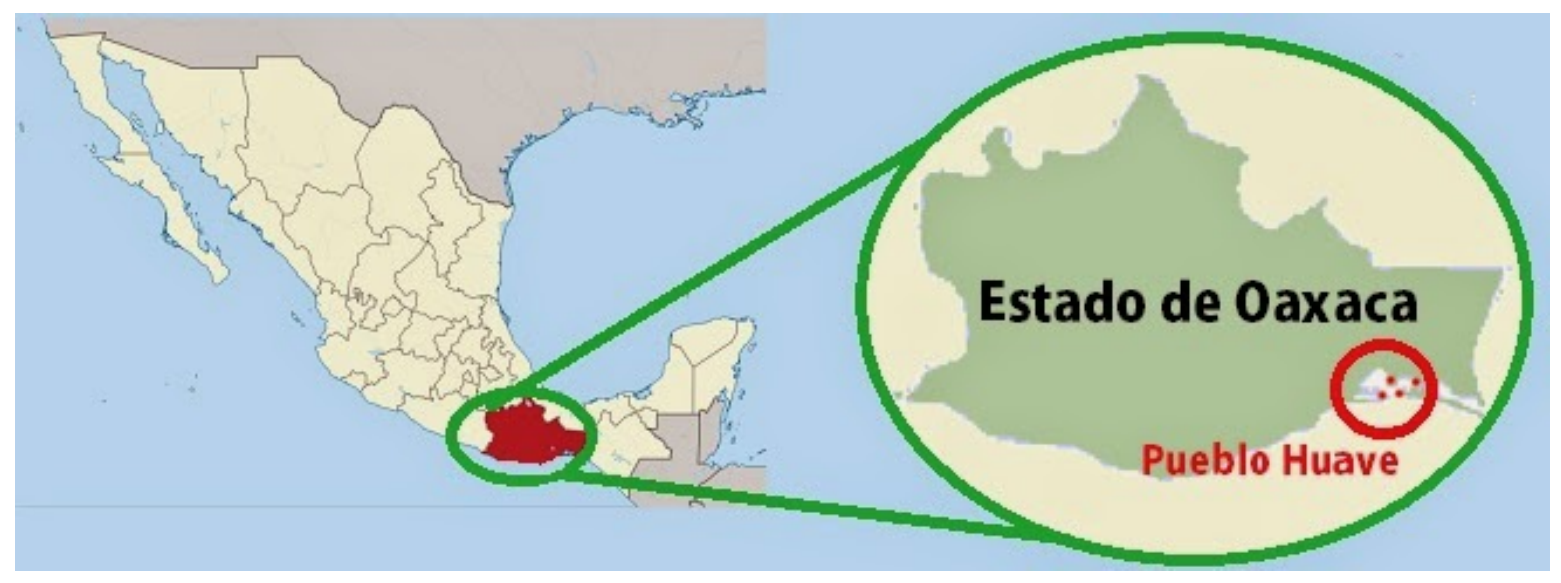

Figura 1. Ubicación de los pueblos ikoots (Fuente: Retomada de https://www.historiacultural.com/2014/10/pueblo-cultura-huave.html).

\footnotetext{
${ }^{3}$ La escuela primaria bilingüe es de organización completa: de primero a sexto existen dos grupos por cada grado. La planta docente consta de 14 profesores, de los cuales 12 están frente a grupo, un profesor de educación física y el director. La mayoría de los docentes son hablantes del huave ‘ombeayiüts'.
} 
Otros autores han señalado que el seudónimo huave en zapoteco significa "mucha gente" (Martínez, 1984). O como sugieren Hernández y Lizama (1996), que es una metamorfosis del patronímico Huazonteco -uno de los primeros asentamientos de este grupo fue precisamente el lugar denominado Huazontlán-, pero aún hoy en día sigue sin aclararse la connotación de la palabra. Actualmente, la cultura ikoots está asentada en San Mateo, San Dionisio, San Francisco y Santa María del Mar. Los tres primeros son municipios ikoots mientras que la última, es una agencia municipal que pertenece a Juchitán de Zaragoza, municipio zapoteco.

Con respecto a la lengua, frente a las 11 familias existentes en territorio mexicano, el ombeayiüts es considerado como una lengua aislada. Esto es debido a que no se ha encontrado su afiliación lingüística con alguna otra familia. Con relación a la vitalidad de la lengua, existen diferencias marcadas en cada uno de los contextos. De acuerdo con datos del INEGI (2010), San Mateo es donde se mantiene el ombeayiüts con mayor vitalidad (86.6 \%), mientras que San Dionisio solamente el 47 \% son hablantes de la lengua, en contraste con Santa María y San Francisco con 17.3 \% y 14.6 \% respectivamente, lo que indica que, en éstos últimos contextos, el fenómeno del desplazamiento lingüístico del español hacia el ombeayiüts ha avanzado de forma acelerada.

\section{Metodología de investigación}

En esta investigación, se adoptó una perspectiva cualitativa para el análisis de los avances o dificultades que muestran los educandos en la producción escrita en ambas lenguas. La revisión y análisis de las producciones escritas en español, se realizó a través de los niveles o hipótesis de la lengua escrita propuestos por Ferreiro y Teberosky (2007):

- Nivel presilábico: la escritura es ajena a toda búsqueda de grafías y sonidos convencionales.

- Nivel silábico: los elementos corresponden a las palabras escritas se intenta una correspondencia entre letras y grafías.

- Nivel alfabético: son escrituras formadas con base en una correspondencia entre fonemas y grafías lo que no se excluyen errores ocasionales. 
Complementado con las aportaciones de Hachen (2002), donde en el nivel alfabético, el autor lo subdivide en tres aspectos más:

Nivel alfabético inicial: consolidación del esquema Consonante-Vocal (CV) para todas las sílabas: MATECA por ma(n)teca, o TOTUGA por to(r)tuga.

Nivel alfabético medio: proyección en el seno de la rima y/o en el ataque de la hipótesis de cantidad generando la posibilidad de otras realizaciones silábicas Consonante-VocalConsonante/Consonante-Consonante-Vocal (CVC-CCV).

Nivel alfabético: habilitación de todos los espacios funcionales de la estructura general de la sílaba Consonante-Consonante-Vocal-Consonante-Consonante (CCVCC) y conceptualización de la posibilidad de la existencia diferentes realizaciones silábicas en una misma palabra.

Mientras que, para el análisis de la producción escrita en ombeayiüts, se hizo a través de las transferencias lingüísticas que los educandos hacen con los elementos fonémicos a su disposición, es decir, la relación o la asociación que van haciendo con el templete grafémico que ya conocen o les son familiares.

Para ello, se motivó a los 24 educandos (15 niñas y 9 niños de entre 7 y 8 años de edad) a que participan en el estudio, y que escribieran pequeños textos y/o oraciones en las dos lenguas (español-ombeayiüts) a partir de textos libres, espontáneos motivados por láminas y paisajes derivados de su contexto natural inmediato. Por ejemplo, sobre los animales que habitan el mundo marino. En el caso del español, todos los niños pudieron hacer producciones con variedad de elementos, mientras que, con el ombeayiüts, la producción fue muy limitada. Sin embargo, mostraron diversidad de aspectos de análisis, como los que se presentan en el siguiente apartado. 


\section{Resultados}

\subsection{Apropiación de la escritura en español}

Los niños mantienen características muy particulares en relación a sus avances de apropiación del sistema de escritura, no obstante, manifiestan avances significativos en su alfabetización en ese ámbito a pesar de ser su L2. Este hecho se puede observar en la figura 2, donde se muestra que la mayoría de los niños se encuentran en el nivel alfabético medio.

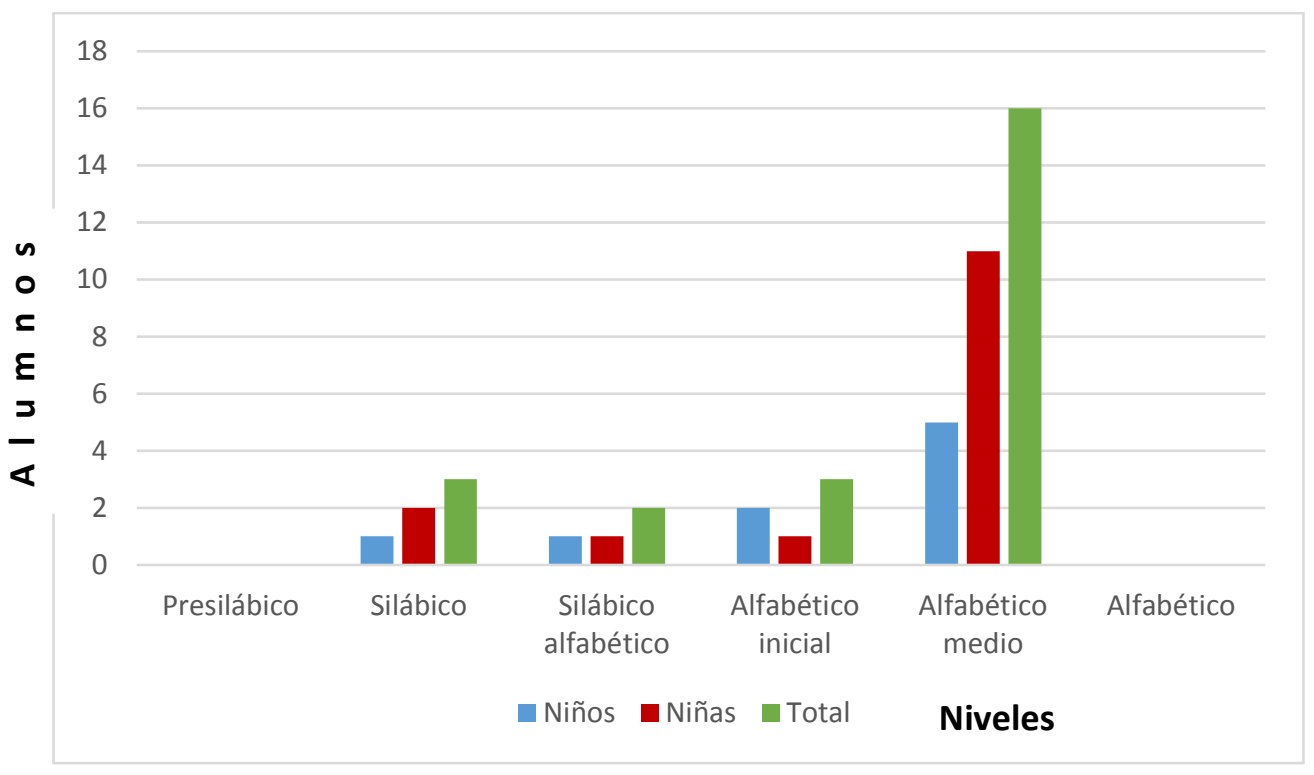

Figura 2. Alfabetización en español.

De acuerdo con estos resultados se puede apreciar que la apropiación de la escritura de los alumnos en español se manifiesta consolidada, ya que, la mayoría se encuentra en el nivel alfabético medio, donde ya dan cuenta en sus escritos del manejo de la composición silábica (CVC-CCV), esto quiere decir, que la mayoría ha superado la concepción silábica CV (Figura 2). 


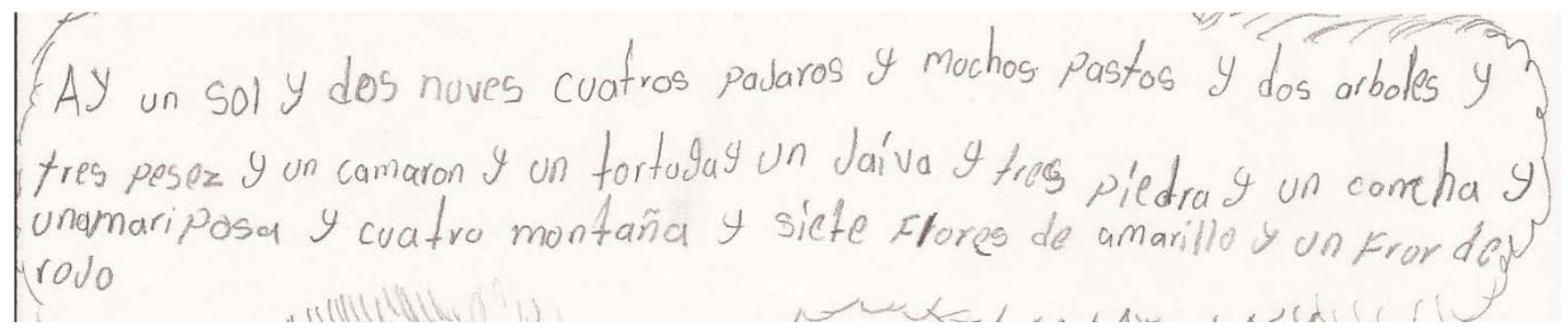

Figura 3. Ejemplo de escritura de la niña M (7 años, 3 meses).

Como se puede apreciar en el escrito de la niña M (Figura 3), hay un manejo apropiado de la construcción y composición de las sílabas de las palabras, es decir, que ya se ha superado en la mayor parte de su escritura la hipótesis de composición CV, evidenciando la existencia de otras realizaciones silábicas que no permiten ser asimiladas por estos esquemas cognitivos. Esto promueve la incorporación de otros segmentos a la sílaba, es decir, la concepción de que la sílaba puede tener más de dos segmentos (Ferreiro y Teberosky, 2007; Hachen, 2002). En el escrito de esta niña, se ve de manifiesto la categorización (CVC) en las palabras "sol, pastos, dos, tres, camarón, tortuga, piedra, concha, montaña” y CCV como se observa en “cuatros, muchos, flores”. Esta característica se ve en otros escritos de los niños, por ejemplo, en palabras como: "cochino, árbol, mar, pescado, pulpo, verdes, hay, otra, alimentación, volando, grandes, chiquitos, viendo, pescado, negras, flacas, comer, miel, gris, cocodrilo” entre otros.

A pesar de que han avanzado en este proceso de apropiación (alfabético medio), aún hay ocasiones en que no se han repuesto todos los espacios funcionales posibles, ni en muchos casos, se ha instaurado la concepción de la coexistencia de diferentes realizaciones silábicas (Hachen, 2002), por ejemplo: pe(s)cato, to(r)tuca, comi(e)ndo, que corresponden al nivel alfabético inicial.

Mientras que los niños que están ubicados en el nivel silábico (3) buscaron una correspondencia sonora entre oralidad y escritura tomando como eje de estructuración la sílaba (una grafía por sílaba). De este modo la sílaba puede aparecer representada por cualquier letra que no implique una correspondencia sonora exacta con la palabra, o bien promover una correspondencia sonora dando lugar a la representación de cada sílaba por la vocal, por ejemplo, en las palabras PCO (pescado), ABL (árbol), AON (camarón). 
En el caso concreto de dos niños, escribieron palabras como NUVS (nubes), TRTGA (tortuga), ARPOSA (mariposa), NANDO (nadando), MOTAAS (montañas), es decir que están ubicados en el nivel silábico alfabético, donde comienzan las hipótesis de cantidad, variedad y posición en por lo menos una de las sílabas.

Por otro lado, también se manifiestan otras características en el escrito de los niños, como la confusión de uso de los grafemas, como se muestra en el siguiente escrito de las niñas A, y B (Figuras 4 y 5).

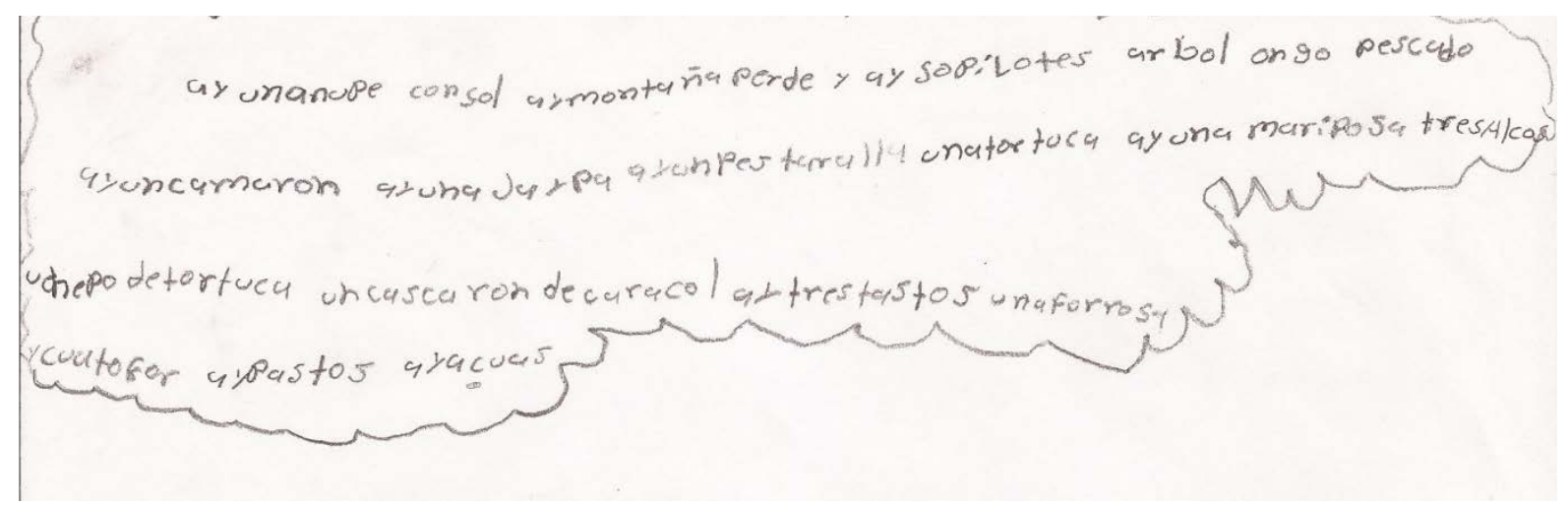

Figura 4. Escritura de A (7 años, 1 mes)

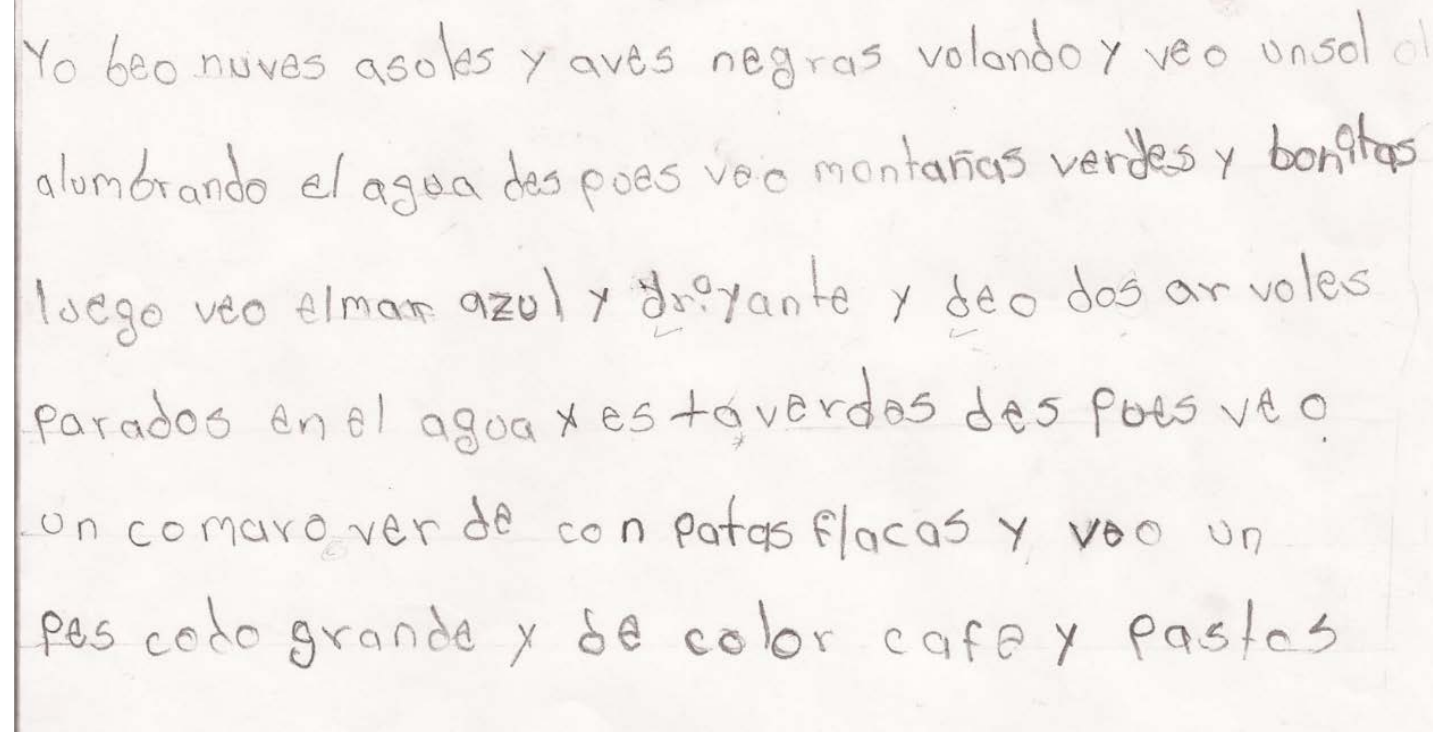

Figura 5. Escritura de B. (7 años, 2 meses)

Ambas presentan una dificultad interesante, que se manifiesta en el uso de los grafemas "p, d, y b”. En el escrito de la niña A (Figura 4), podemos observar una confusión ante la presencia de los grafemas "p y b”, como en las palabras “nupe” en vez de nube, "perde” por 
verde y “jaypa” por jaiba, así como “tortuca” en lugar de tortuga. Se puede predecir que esta niña no logra identificar el sonido de la oclusiva, bilabial, sonora /b/, por eso lo sustituye por la bilabial, sorda "p", con la cual tiene mayor familiaridad. Esto se observa en las palabras "sopilotes, pescado, pes, mariposa, pastos".

Mientras que la niña B (Figura 5), muestra una escritura legible, pero también con dificultades en el manejo de “d y b”. A pesar de tener una familiaridad y reconocimiento del uso y sonorización de dichos grafemas en las palabras que tiene el escrito, también los confunde en dos ocasiones en las palabras “driyante” en lugar de brillante y veo por “deo", aunque ésta última, aparece escrita 6 veces de manera adecuada. Podemos suponer pues que por las características fonológicas de la lengua en cuestión ha tenido alguna dificultad.

Otros niños revelan otras características importantes en sus escritos, como la dificultad para marcar el género o el número, como podemos observar en el siguiente escrito (Figura 6).

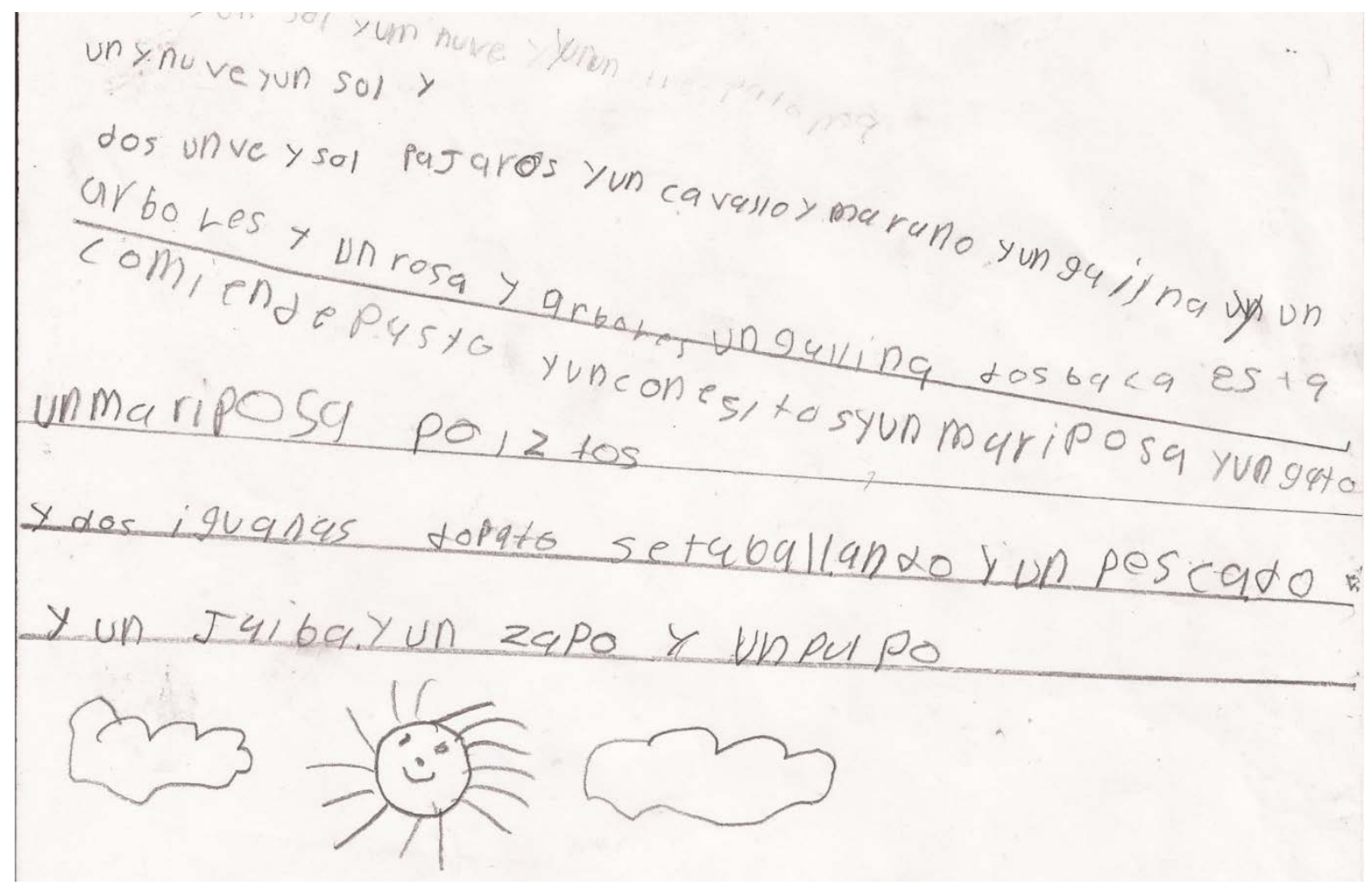

Figura 6. Escritura de la niña F (8 años, 0 meses).

Como se puede apreciar en la Figura 6, la escritura de la niña F muestra muchos fenómenos que comentar, si bien se resaltan aquí sólo algunos de ellos. Es evidente que ha adquirido un nivel medio de alfabetización respecto al conocimiento del sistema de escritura, aunque 
todavía se advierten algunos desaciertos propios en esta etapa, por ejemplo: "marano por marrano, conesito por conejito, poiztos por pollitos, seta por está, ballando por bañando”. Asimismo, se resalta en el escrito que comúnmente confunde el uso de “un”, ya que lo considera como un marcador de cantidad que acompaña a todo nombre o sustantivo, sin considerar la relación con el género o número, como se aprecia en las frases "un nuve, un caballo, un gallina, un arboles, un rosa, un gato, un mariposa, un pescado, un jaiba, un zapo”. Este mismo fenómeno ocurre en el escrito de otros niños con palabras como las siguientes: una sol, una caballo, una marano, una gallina, una baca, una pescado, una pulpo, una cangrejo, una sapo, una pidra, una jum (cocodrilo), una arbol; para ellos no hay diferencia para escribir “un o una” sino una sola opción.

Una de las explicaciones para dicha situación, es el hecho de que en la lengua ombeayiüts (su L1) no existe artículo marcador de género, sino que se ocupa la palabra aaga como marcador neutro o como determinante, es decir, se ocupa en todos los contextos de una oración sin hacer diferencia de masculino o femenino, por ejemplo: aaga naxey ‘el señor’ y aaga najtaj 'la señora', aaga win 'la tortuga' y aaga pet 'el perro'.

De igual forma, debido al nivel académico en que se encuentran los niños, en sus escritos se manifiestan desaciertos en el manejo de fonemas y grafemas, pero también se asume que la forma o método de alfabetización que retoma el docente tiene influencia en ello. Para este caso, el método onomatopéyico se enfrenta a dificultades, ya que en español se dan los siguientes fenómenos:

- Un grafema que no corresponde con ningún fonema, como la $\mathbf{h}$.

- Un grafema que aparece como marca diferenciadora respecto a la pronunciación de un grafema, como es el caso de $\boldsymbol{u}$ en gue y en gui, pero que no funciona allí como notación de fonema, es decir que "no suena”.

- El mismo grafema u que es obligatorio junto a la q, como en queso y quiso, pero que, como en el caso anterior, no funciona como fonema.

- La existencia de dos grafías para permitir la notación de un solo fonema como en el caso de ll, rr.

- La existencia de un solo grafema para permitir la notación de dos fonemas como en el caso de $\mathbf{x}$. 
- La posibilidad de que un mismo fonema aparezca en la escritura relacionado con grafemas diferentes, como en los casos b/v, s/c/z, entre otros (Desinano, 2002).

Aunque esto se debe ir superando a lo largo de la escolaridad primaria, en ocasiones no es atendido o retomado por los docentes, por eso es menester que se considere para ir consolidando el proceso de apropiación de la escritura por parte de los alumnos y poder llegar al nivel alfabético. Con respecto a la primera lengua, también se encontraron fenómenos y situaciones interesantes.

\section{2 . Apropiación de la escritura en ombeayiüts}

Como ya se ha hecho referencia, los niños de segundo grado tienen como primera lengua al ombeayiüts y les es difícil la comprensión del español, sin embargo son alfabetizados en esta lengua. A partir de la descripción de elementos de su contexto inmediato, los educandos produjeron pequeñas oraciones y en algunos casos solo palabras. La finalidad de la descripción fue, que a partir de la producción escrita de los alumnos, realizar la comparación entre los fonemas compartidos entre el español y el ombeayiüts ${ }^{4}$ y las exclusivas de la lengua materna. Aunque la producción escrita de los alumnos fue limitada, mostraron elementos lingüísticos y de transferencia lingüística interesantes para el análisis.

\subsubsection{Fonemas consonánticos compartidos con el español}

La lengua ombeayiüts comparte con el español los siguientes fonemas con sus respectivas grafemas, como son: /p/, /t/, /m/, /n/, /r/, /l/, /r/, /tg/, entre otros, de los cuales /k/, /s/ son de escritura inestable ${ }^{5}$. En éstos, dentro de las descripciones que se realizaron, se presentaron ciertas dificultades al momento de su escritura, mientras que en los de escritura estable sólo hubo problema en dos casos: en sílabas cerradas. Podemos observar los ejemplos en la tabla 1.

\footnotetext{
${ }^{4}$ Para este trabajo se utilizaron los siguientes fonemas del ombeayiüts de acuerdo a los avances en relación a las discusiones para la normalización de la lengua realizadas por el Instituto Nacional de Lenguas Indígenas

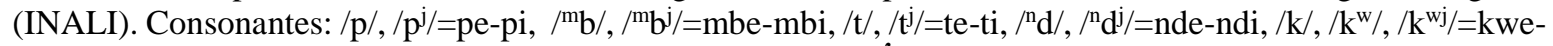

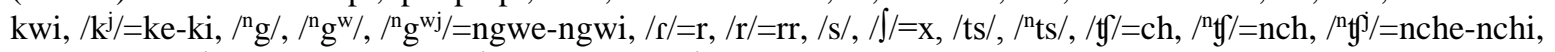

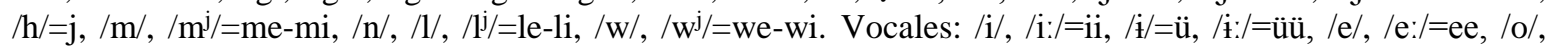
/o:/=oo, /a/, /a:/=aa.

${ }^{5}$ Por inestable nos referimos a aquellos fonemas que pueden ser representados por varios grafemas y que en lenguas originarias no se reducen a problemas de ortografía, sino a la falta de acuerdos para representarlos, aunque la tendencia es a usar un solo grafema. Este es el caso de las compartidas k (k, c, qu) y s (s, z).
} 
Tabla 1. Fonemas compartidos con el español.

\begin{tabular}{|c|c|c|c|c|c|c|}
\hline \multirow[t]{2}{*}{$\mathbf{N} / \mathbf{P}$} & \multirow{2}{*}{$\begin{array}{c}\mathrm{p} / \\
\text { “potwit” }\end{array}$} & \multicolumn{2}{|c|}{$/ \mathrm{t} /$} & \multirow{2}{*}{\multicolumn{2}{|c|}{$/ \mathbf{m} /$}} & \multirow{2}{*}{$\begin{array}{c}/ \mathbf{n} / \\
\text { "najaw" }\end{array}$} \\
\hline & & küt & "tixem" & & & \\
\hline 01 & potwit & kait & tixem & mil & tixem & najaü \\
\hline 02 & & cuet & & & & najaw \\
\hline 03 & pikiw & & & mil & ticen & nagaw \\
\hline 04 & popllit & kuc & tixem & & tixem & \\
\hline 05 & potwit & kuet & & & & najaw \\
\hline 06 & & cuc & & & tixen & \\
\hline 07 & potllit & quec & & mil & & najaw \\
\hline 08 & & Cuc & tixem & & tixem & \\
\hline 09 & potllit & cut & & & & najaw \\
\hline 10 & & & tixen & mil & tixen & \\
\hline 11 & potfit & quet & & & & \\
\hline 12 & & kuc & & & & \\
\hline 13 & popllit & kuec & tisem & mil & tisem & najai \\
\hline 14 & pomik & cuem & tixiem & mil & tixiem & najaw \\
\hline 15 & & quet & ticem & & ticem & \\
\hline 16 & popllit & cuc & tixem & mil & tixem & \\
\hline 17 & & cat & & & & najao \\
\hline
\end{tabular}

Como se observa en esta tabla, los niños ya mantienen cierto conocimiento acerca de los grafemas compartidos con el español, de ahí que realicen la transferencia para la escritura de su lengua.

Para la palabra potwit 'zopilote', se refleja una buena transferencia de los educandos con relación al fonema /p/, porque todos lo utilizaron de manera adecuada en inicio de palabra. Pero en la primera sílaba pot, se visualiza que dos de los niños (04 y 16) cambian el último grafema “t”, una oclusiva, dental sorda, por la “p” que también es oclusiva, bilabial y sorda. Dichas características pudieron provocar la confusión, ya que solo cambian en el punto de articulación, esto a pesar de que han tenido acercamiento con sus experiencias en español. 
Pero cuando el fonema /t/ aparece en inicio de palabra, como en el ejemplo de la palabra tixem 'camarón' los niños también hacen una buena interpretación y transferencia del fonema. Aunque como ya se mencionó, cuando el fonema /t/ aparece al final de palabra, como en el ejemplo de küt 'pescado', existe una dificultad para los niños, donde se apreciará una sustitución por el grafema “c”, esto se ve reflejado en siete circunstancias, o con “m” en una.

El mismo fenómeno ocurre con el fonema /m/: cuando se encuentra al inicio de palabra, no existe confusión alguna para los niños, hacen una correspondencia adecuada, así lo manifiestan con la palabra mil 'pez lisa'. Sin embargo, cuando se encuentra al final de sílaba, como en el caso de tixem, tres de los niños (3, 6 y 9) lo cambiaron por el fonema /n/, es decir, una nasal, alveolar, sonora, la diferencia con el fonema anterior es en relación al punto de articulación, una es alveolar y la otra bilabial (Tabla 1)..

Otro de los fonemas compartidos con el español y de escritura estable que se encontró en el escrito de los niños es la /n/. En este caso es notable que existe una escritura adecuada, donde los niños reconocen el sonido del fonema /n/ cuando se encuentra a principio de palabra al igual que las anteriores. Entonces se puede decir que en relación a los fonemas consonánticos compartidos con el español y de escritura estable, los niños muestran un buen conocimiento, porque hacen una transferencia adecuada. La dificultad aparece para algunos cuando aparecen al final de palabra.

\subsubsection{Fonemas vocálicos}

Asimismo, existe una buena transferencia en cuanto a las vocales, que podemos observar en la tabla 2.

Las cuatro vocales que se comparten con el español: “a, e, i, o”, son también de escritura estable, pero en el cuadro anterior sólo se hace mención de tres, ya que, son las que más aparecen en los escritos de los niños. 
Tabla 2. Fonemas vocálicos registrados.

\begin{tabular}{|c|c|c|c|c|}
\hline \multirow[t]{2}{*}{$\mathbf{N} / \mathbf{P}$} & \multicolumn{2}{|c|}{$/ \mathbf{a} /$} & \multirow{2}{*}{$\begin{array}{c}\text { /i/ } \\
\text { ijkyaw } \\
\text { (dos) }\end{array}$} & \multirow{2}{*}{$\begin{array}{c}/ \mathbf{0} \\
\text { oik } \\
\text { (nube }\end{array}$} \\
\hline & apikiw & najaw & & \\
\hline 01 & apiki & najaü & ijkiaw & oc \\
\hline 02 & apiki & & & oc \\
\hline 03 & apikiw & najaw & ijkiaw & \\
\hline 04 & apikiw & nagaw & & oik \\
\hline 05 & & & ijquiaw & oqui \\
\hline 06 & apikiw & najaw & ijkiaw & oik \\
\hline 07 & apikiw & najaw & ijciaw & oik \\
\hline 08 & apikiw & & & oit \\
\hline 09 & apikiw & najaw & ijkiaw & oc \\
\hline 10 & apik & & & oic \\
\hline 11 & & & ijquiaw & oic \\
\hline 12 & apikiw & & ijcaw & \\
\hline 13 & apikiw & najaw & & oxi \\
\hline 14 & apiki & najaw & ijkia & oxi \\
\hline 15 & & & & oqui \\
\hline 16 & apikiw & najao & iquiaw & oic \\
\hline 17 & apikiw & & ijkiaw & oik \\
\hline
\end{tabular}

El grafema "a" es constante, existe un reconocimiento por parte de los educandos, como se muestra cuando es usado a principio de palabra como en caso de apikiw 'cuatro'; así mismo cuando aparece a final o a mitad de sílaba como en na-jaw 'ver/mirar'. Pasa lo mismo con la vocal /i/, al inicio y mitad de sílaba, como lo ejemplifica la palabra ijkiaw ‘dos’, así también con la vocal /o/. Esto indica nuevamente que la transferencia de los fonemas compartidos con el español que tienen escritura estable se da de manera apropiada, tanto en las consonantes como en las vocales. 


\subsubsection{Consonantes compartidas inestables}

Como estaba previsto, se presentan dificultades en las consonantes compartidas que en español tienen escritura inestable. Podemos ver los resultados en la tabla 3.

Tabla 3. Consonantes inestables.

\begin{tabular}{ccc}
\hline $\mathbf{N} / \mathbf{P}$ & $\begin{array}{c}\text { /k/ } \\
\text { "küt” }\end{array}$ & $\begin{array}{c}/ \mathbf{s} / \\
\text { “saxom” }\end{array}$ \\
\hline 01 & kait & Zaxiüm \\
02 & cuet & Sasiom \\
03 & & Saxom \\
04 & kuc & Zaciom \\
05 & kuet & Saxom \\
06 & cuc & \\
07 & quec & Sasiom \\
08 & & Zaxiom \\
09 & cut & Xasom \\
10 & quet & Saciom \\
11 & & Sasiom \\
12 & cuem & Sasion \\
13 & quet & Zaciom \\
14 & kuc & \\
15 & cat & Xasiom \\
16 & quet & \\
\hline & & \\
\hline
\end{tabular}

A partir de los resultados obtenidos (Tabla 3), se pueden hacer varias reflexiones. Como se ha mencionado, en español el fonema / $\mathrm{k} /$ tiene escritura inestable, y esto es lo que reflejan a primera vista los niños. Para escribir la palabra küt 'pez/pescado', hacen uso tanto de 'k, c y qu”, esto denota que no distinguen que grafema emplear. Esto tiene que ver con la familiarización y la asociación de sonidos que los niños realizan, porque la “c y qu” tienen mayor uso y práctica en el español que la "k", no así en el ombeayiüts, que solamente se utiliza la "k". Esta característica es muy común en el escrito de los alumnos y se vio reflejada 
también en palabras como quiec, quec 'pájaro', condo 'después', cawex 'arriba', can 'piedra'.

Sucede algo similar con la palabra saxom 'encontrar'. La confusión aparece en el uso del grafema “s” donde los alumnos lo utilizan con “z” y "s”. Es interesante observar que otros invierten la sílaba utilizando la “x” que en ombeayiüts se utiliza para la fricativa, posalveolar, sorda $/ \mathrm{J} /$, esto se aborda en el siguiente apartado.

\subsubsection{Consonantes simples exclusivas del ombeayiüts}

La lengua ombeayiüts, tiene un total de 17 consonantes exclusivas, pero se presentan y analizan únicamente las que aparecen con los escritos de los niños (obsérvese la tabla 4).

Como se puede apreciar en la tabla 4, el primer fonema / $/$, una fricativa postalveolar, sorda, cuando aparece al principio de palabra y de sílaba como en xike ‘yo’ (13/16) y en tixem ‘camarón’ (6/7), la mayoría de los educandos ya tiene una asociación de sonido muy importante con el grafema “x”, y lo utilizan de manera adecuada. No obstante, también hubo tres niños (3/16) en xike que lo cambiaron por la fricativa, alveolar, sorda /s/. El cambio puede deberse a la proximidad de los puntos de articulación, ya que uno es alveolar y la otra postalveolar, lo que pudo conducir a que los niños asocien los sonidos cuando no tienen un grafema para este fonema específico, mientras que para tixem solo un niño (1/7) lo confundió con el grafema "c".

Pero cuando la “x” se da en proximidad del fonema /s/ como en el caso de saxom 'encontrar', aparece una mayor dificultad y lo escriben con “s (7/14), c (3/14) y x (4/14)”. Los niños asumen que existe un sonido distinto en xom, pero en realidad en términos lingüísticos se considera que la “ $\mathrm{x}$ ” es la realización de $/ \mathrm{s}^{\mathrm{j}} /$, y puede deberse a eso la confusión. Además, que el uso y sonido de la “ $x$ ” es confuso en español, ya que puede sonar como en Oaxaca y México, o como en Xochimilco, mientras que en el ombeayiüts es utilizado únicamente como “x”, y en este contexto incluso, se manifiesta casos de inversión como en xasom (3/14) con los niños $(09,15$ y 16). 
Tabla 4. Consonantes simples exclusivas del ombeayiüts.

\begin{tabular}{|c|c|c|c|c|c|c|c|c|}
\hline \multirow[t]{2}{*}{$\mathbf{N} / \mathbf{P}$} & \multicolumn{3}{|c|}{ /]/ “x” } & \multicolumn{3}{|c|}{ /ü/ “ü” } & \multicolumn{2}{|c|}{ /w/ “w” } \\
\hline & xike & saxom & tixem & myük & xyül & küt & ijkyaw & portwit \\
\hline 01 & xique & zaxiüm & tixem & miek & xiel & kait & ijkiaw & potwit \\
\hline 02 & xique & sasiom & & miek & siel & cuet & ijkiaw & \\
\hline 03 & xique & saxom & & & & & & \\
\hline 04 & xique & zaciom & tixem & miek & ziel & kuc & ijquiaw & popllit \\
\hline 05 & xike & saxom & & miek & xiel & kuet & ijkiaw & potwit \\
\hline 06 & xique & & & miec & siel & CUC & & \\
\hline 07 & xique & sasiom & & & ziel & quec & ijciaw & potllit \\
\hline 08 & & zaxiom & tixem & & sul & & & \\
\hline 09 & xique & xasom & & miek & xiel & cut & ijkiaw & potllit \\
\hline 10 & sique & saciom & & & xiel & quet & ijquiaw & potfit \\
\hline 11 & sique & & & miec & siel & & ijcaw & \\
\hline 12 & xique & sasiom & tixem & & & & & popllit \\
\hline 13 & xique & sasion & tixiem & miek & ziel & cuem & & pomik \\
\hline 14 & xique & zaciom & ticem & & & quet & & \\
\hline 15 & & xasom & & & xiel & & & \\
\hline 16 & sique & xasiom & & miec & ciel & cat & iquiaw & \\
\hline 17 & xique & & & & ciel & & & \\
\hline 18 & xike & & tixiem & miec & ciel & quet & ijkiaw & \\
\hline
\end{tabular}

Para el fonema "ü" hay una mayor dificultad para su uso. A diferencia de las vocales compartidas los niños le dan diversas interpretaciones. Por ejemplo, en el contexto de las palatalizadas, automáticamente le asignan el grafema "e" como lo muestra la tabla 4 en las palabras miek ‘mariposa' (10/10), xiel ‘árbol/palo' (14/15)” y solo un niño (1/15) le asigna 
"u”. Pero en las palabras precedidas por el grafema "k", mantienen también diversas interpretaciones como se muestra en la palabra küt, donde los niños lo interpretan como "ue” (7/12), mientras que otros lo escribieron con " $u$ ” (3/12), así como con “ai” (1/12) y “a” (1/12); esto demuestra que existe una consciencia sobre el sonido de la /ü/, es decir, que se dan cuenta de que existe un sonido diferente, pero debido a la familiaridad con la escritura del español optan por "e" o por "u".

Para el uso de la aproximante, labiovelar, sonora /w/ los educandos lo escriben y asocian el sonido de manera apropiada al final de sílaba, como en el caso de la palabra ijkiaw 'dos' (10/10), mientras que a mitad de palabra y en principio de sílaba como en pot-wit 'zapilote' se da la sustitución por la aproximante, palatal, sonora /j/ escrita con "Il” (4/8), así como con la bilabial /m/ (1/8) y por la fricativa, labiodental "f” (1/8) y dos lo escribieron de manera apropiada (2/8).

Una situación a resaltar en esta misma palabra ijkiaw, es con relación al uso de la fricativa, glotal, sorda /h/; los niños lo utilizan de forma adecuada, a diferencia en la palabra ndoj 'después’ del mismo cuadro. En esta palabra aparece a final de sílaba; puede ser que, en esta circunstancia, al estar después de la vocal "i” el sonido se escucha más marcado y no deja espacio para la confusión con otros sonidos.

\subsubsection{Consonantes modificadas exclusivas del ombeayiüts}

La lengua ombeayiüts tiene una serie de consonantes modificadas, pero la tabla 5 solamente muestra lo que representaron los niños.

Para la escritura de "nd" y "mb" se puede apreciar que la palabra "ndoj”, donde se hace uso del fonema $/{ }^{\mathrm{n}} \mathrm{d} /$, todos los niños lo escribieron iniciando únicamente con el grafema "d" y omitiendo la parte nasalizada. Esto muestra el desconocimiento de los niños de los grafemas del ombeayiüts dado únicamente transfieren lo conocido por ellos. Lo que se utiliza en español, es la "d”, mientras que en el ombeayiüts nunca se da al inicio de palabra, solamente con das 'danza' como préstamo del español, y si aparece a final de palabra cuando hay vocales alargadas, como en eed 'pluma', need 'ladrón', piid 'epazote', iid 'excremento', meed 'huipil', ncheed 'ombligo de pescado/bejuco para preparar atole de espuma', entre otros. Pero lógicamente los niños no tienen consciencia de este proceso. 
Tabla 5. Consonantes modificadas.

\begin{tabular}{ccc}
\hline $\mathbf{N} / \mathbf{P}$ & $\begin{array}{c}\text { /nd/ } \\
\text { “ndoj” (después) }\end{array}$ & $\begin{array}{c}\text { /'b/ } \\
\text { “mbaj” (flor) }\end{array}$ \\
\hline 01 & do & ba \\
02 & dow & baj \\
03 & do & baj \\
04 & doj & baj \\
05 & do & mbaj \\
06 & doj & ba \\
07 & do & ba \\
08 & & baj \\
09 & doj & \\
10 & do & baj \\
11 & do & ba \\
12 & doj & ba \\
13 & & ba \\
14 & do & baj \\
15 & doj & ba \\
16 & & baj \\
17 & do & \\
\hline
\end{tabular}

Otra situación a resaltar, es referente a la terminación de la misma palabra ndoj 'después'. Únicamente cinco niños (5/14) escribieron con el grafema utilizado para representar la fricativa, glotal, sorda /h/, mientras que ocho (8/14) no lo hicieron, es decir, solo escribieron “do” y uno (1/14) lo sustituyó por la “w”. Esto puede indicar que estos niños no reconocen dicho fonema, o si lo hacen, aún no logran saber cómo representarlo, a pesar de que en español han tenido familiaridad con / $\mathrm{x}$ / la fricativa, velar, sorda que se escribe “j”. Esta tiene un sonido similar, el cambio ocurre en el punto de articulación. Aunque en el español su contexto de escritura es inestable con la “g”, “x” y “j”, por ejemplo: Juan, jarabe, jamón, jícara, gis, gelatina, joroba, jugar, raja, caja, bajo, Xalapa, entre otros, y casi no aparece al 
final, mientras que en el ombeayiüts aparece tanto a principio como a final de sílaba para representar el fonema $/ \mathrm{h} /$.

Por otro lado, en lo que respecta a la palabra mbaj 'flor', el fonema /mb/ pertenece al grupo de las nasales, en la que nuevamente el desconocimiento incide sobre la transferencia de sus habilidades de escritura. La mayoría escribió iniciando con /b/ (14/15), pero esta oclusiva, bilabial, sonora, no aparece comúnmente a inicio de palabra en ombeayiüts, sino al final igual que la [d], después de vocales alargadas, como en: loob, naab, poob, cheeb, saab, roob, etc. Esto tiene que ver que, en este contexto, las oclusivas, bilabial y dental, sordas, se sonorizan y por eso se convierten en [b] y [d]. Aunque también se puede apreciar que un niño sí utilizó la “mb” en el escrito.

\section{Conclusiones}

En el proceso de apropiación de la escritura es importante observar y analizar los fenómenos que manifiestan los alumnos en sus escritos. Pero más significativo aún, como dice Ferreiro "además de la mano que hace trazados con el lápiz, del ojo que discrimina formas, y de la boca que emite sonidos, hay un sujeto que piensa", es al que hay que atender y apoyar en su proceso, y habría que hacerlo a partir de su primera lengua, y en un segundo momento, desarrollar la segunda lengua para encaminarse a un bilingüismo eficiente.

En el estudio que presentamos, los niños ikoots son alfabetizados de forma directa en la L2 el español- sin anteponer su L1. Sin embargo, en sus producciones en español, la mayoría de los niños se encuentra en el nivel alfabético medio, es decir, que ya existe una apropiación de la composición silábica (CVC-CCV), lo que indica que estos niños han superado la concepción silábica CV. A pesar de que han avanzado en este proceso de fonetización (alfabético medio), aún hay ocasiones en que no se han repuesto todos los espacios funcionales posibles. Los niños que se ubicaron en el nivel silábico, se puede decir que buscaron una correspondencia sonora entre oralidad y escritura, tomando como eje de estructuración la sílaba (una grafía por sílaba).

Mientras que en ombeayiüts se puede considerar lo siguiente: los educandos se apoyan de los elementos fonémicos que le son familiares (en español) y los transfieren a su L1. Por ello, con los fonemas consonánticos y vocálicos compartidos con el español y de escritura estable, realizan una buena transferencia, esto quiere decir, que tienen conocimiento sobre su uso. No obstante, también aparecen dificultades, por ejemplo, cuando dichos fonemas aparecen a 
final de palabra. Para los de escritura inestable, como es el caso de “k” y “s”, los niños reflejan hipótesis interesantes porque anteponen el fono, pero manifiestan diversidad en la representación como en el caso del grafema " $k$ " hicieron uso de (k, c y qu) y con la "s" (z y s) indistintamente. Los alumnos hacen una transferencia a partir de sus repertorios lingüísticos conocidos o apropiados, es decir, utilizan los grafemas usados en el español para representar la escritura de su lengua materna.

Para la transferencia de las consonantes exclusivas del ombeayiüts, los niños muestran en algunos casos la falta de familiaridad con éstos, pero también el conocimiento de otros. Por citar el ejemplo del fonema fricativo, posalveolar, sordo / $/$ /, donde a principio de sílaba las dificultades son mínimas, pero la dificultad surge cuando este mismo fonema se encuentra en proximidad del fonema /s/. Al fonema /ì/, los niños le asignan diversas interpretaciones, al igual que los grupos consonánticos “nd y mb” sólo hacen uso del grafema “d y b” aunque los dos no existen a principio de sílaba en el ombeayiüts, pero en español sí. Sin duda, el desarrollo de estudios relativos a la adquisición de la escritura de niños y niñas bilingües en diferentes contextos es una línea de investigación que merece la atención de la comunidad científica. 


\section{Referencias}

Agustín, M. (2010). An overview of variables affecting lexical transfer in writing: A review study. International Journal of Linguistics, 2(1), 1-17. DOI: 10.5296/ijl.v2i1.445

Cummins, J. (1983). La competencia lingüística y el rendimiento académico. En Juan Oller (ed.), Problemas en la investigación de la enseñanza de idiomas. Rowley, Massachusetts: Newbury house.

Cummins, J. (2001). ¿Qué sabemos de la educación bilingüe? Perspectivas psicolingüísticas y sociológicas. Revista de educación, 326, 37-61.

Desinano, N. (2002). Didáctica de la lengua para el $1^{e r}$ ciclo E.G.B. Rosario, Argentina: Homo Sapiens.

Ferreiro, E. (2004). Alfabetización. Teoría y Práctica. México: Siglo XXI.

Ferreiro, E. y Gómez, M. (1991). Nuevas perspectivas sobre los procesos de lectura y escritura. Buenos Aires, Argentina: Siglo Veintiuno editores.

Ferreiro, E. y Teberosky, A. (2007). Los sistemas de escritura en el desarrollo del niño. (23 ${ }^{\mathrm{a}}$ ed.). México: Siglo XXI.

Francis, N. y Hamel, R. (1992). La redacción en dos lenguas: escritura y narrativa en tres escuelas bilingües del Valle del Mezquital. Revista Latinoamericana de Estudios Educativos, 22(4), 11-35.

Gómez, M. (2000). El proceso de adquisición del sistema de escritura. En M. Gómez y A. Martínez (Coords.), La adquisición de la lectura y la escritura en la escuela primaria (pp. 135-140). México: SEP.

Gómez, E. (2005). Diagnóstico regional del istmo de Tehuantepec. Proyecto Perfiles Indígenas. CIESAS Istmo

Hachen, R. (2002). Conocimiento lingüístico y reflexión metalinüística. El rol de las conceptualizaciones en torno a la estructura de la sílaba en el proceso de alfabetización. Lectura y Vida, 23 (2), 1-14.

Halliday, M. (1989). Spoken and written language. Nueva York: Oxford University Press.

Hernández, J. y Lizama, J. (1996). Cultura e identidad étnica en la región huave. Oaxaca: UABJO 
Instituto Nacional de Estadística y Geografía [INEGI]. (2010). Censo de población y vivienda. México: $\quad$ INEGI. https://www.inegi.org.mx/programas/ccpv/2010/

Instituto Nacional para la Evaluación de la Educación [INEE] (2017). Directrices para mejorar la atención educativa de niñas, niños y adolescentes indígenas. México: INEE.

Krashen, S. (1999). Condemned without a trial: Bogus arguments against bilingual education. Portsmouth, NH: Heinemann.

León, N. (1903). Catálogo de antigüedades huavis del estado de Oaxaca existentes en el Museo Nacional de México. México: Imprenta del Museo Nacional.

Lerner, D. (2003). Leer y escribir en la escuela: lo real, lo posible y lo necesario. México: SEP/Fondo de Cultura Económica.

Marsh, D., Pérez, M. y, Ráez, J. (2015). CLIL in Action: Voices from the Classroom. Newcastle upon Tyne: Cambridge Scholars.

Martínez, M. (1984). Los huaves. México: Archivo General del Gobierno del Estado de Oaxaca

Nemirovsky, M. (1999). Sobre la enseñanza del lenguaje escrito...y temas aledaños. México: Paidós.

Odlin, T. (1989). Language transfer: Cross-linguistic influence in language learning. Cambridge: Cambridge University Press.

Ong, W. (2016). Oralidad y escritura. Primera edición electrónica. México: FCE.

Pérez, M. (2012). Clil research in Europe: Past, present, and future. International Journal of Bilingual Education and Bilingualism, 15 (3), 315-341.

Prado, J. (2004). Didáctica de la lengua y la literatura para educar en el siglo XXI. Madrid, España: Editorial La Muralla

Schmelkes, S. (2002). La enseñanza de la lectura y la escritura en contextos multiculturales. Conferencia presentada en el VII Congreso Latinoamericano para el Desarrollo de la Lectura y la Escritura, Puebla, México. Recuperado de www.amdh.com.mx/ocpi/documentos/ docs/6/15.doc

Signorini, I. (1991). Los huaves de San Mateo del Mar. Ideología e instituciones sociales. México: INI 
Vigil, N. (2004). Acciones para desarrollar la escritura en lenguas indígenas. Glosas didácticas, 12, 174-183. 